\title{
Cellular heterogeneity in brown adipose tissue
}

\author{
Yasuo Oguri ${ }^{1,2,3}$ and Shingo Kajimura, ${ }^{1,2,3}$ \\ 'UCSF Diabetes Center, San Francisco, California, USA. ${ }^{2}$ Eli and Edythe Broad Center of Regeneration Medicine and Stem Cell Research, San Francisco, California, USA. ${ }^{3}$ Department of Cell and Tissue Biology, \\ UCSF, San Francisco, California, USA.
}

\begin{abstract}
Brown adipose tissue (BAT) contains mitochondria-enriched thermogenic fat cells (brown adipocytes) that play a crucial role in the regulation of energy metabolism and systemic glucose homeostasis. It was presumed that brown adipocytes are composed of a homogeneous cell population. In this issue of the $J C I$, however, Song and colleagues report a previously uncharacterized subpopulation of brown adipocytes that display distinct characteristics from the conventional brown adipocytes in their molecular signature, regulation, and fuel utilization. The present study provides novel insight into our understanding of cellular heterogeneity in adipose tissues.
\end{abstract}

\section{Roles of brown adipose tissue} Brown adipose tissue (BAT) contains numerous brown adipocytes with highly enriched mitochondria and multilocular lipid droplets, and possesses the remarkable capacity to dissipate energy in the form of heat. Thus, BAT has been viewed merely as a thermogenic organ for a long time; however, emerging evidence suggests that the role of BAT is far more than thermogenesis (1). As an example, BAT functions as a significant metabolic sink for glucose, fatty acids, and branchedchain amino acids (BCAAs), such that activation of BAT leads to a profound improvement in systemic glucose homeostasis, lipid homeostasis, and BCAA clearance in rodents and humans (2-4). Accordingly, BAT biology has become a significant area of research in the field of metabolic disorders beyond obesity because a better understanding of this organ may lead to new interventions to improve metabolic health, such as insulin resistance, dyslipidemia, and type 2 diabetes (5).

\section{Adipose cell heterogeneity - more than brown, beige, and white}

The adipose tissue was once thought to be a monolithic organ that was composed of homogeneous adipose cell populations. However, it is becoming clear that each adipose tissue depot contains a variety of fat cells, including brown adipocytes, beige adipocytes, and white adipocytes, that arise from distinct developmental origins $(6,7)$. Even within an adipose tissue depot, recent studies indicate the existence of heterogeneous cell populations. For instance, Chen et al. recently described a previously uncharacterized type of beige adipocyte whose biogenesis is regulated independently from the canonical $\beta 3$ adrenergic receptor ( $\beta 3-\mathrm{AR}$ ) signaling (8). This beige fat population stems from a distinct pool of progenitor cells and possesses a unique fuel utilization (e.g., glycolytic metabolism as opposed to fatty acid oxidation), and thus, these cells are termed glycolytic beige fat (or g-beige fat). Relative to beige adipocytes, brown adipocytes in the interscapular BAT region were thought to be homogeneous, although Cinti's group has previously reported that BAT contained two types of brown adipocytes that expressed high or low levels of uncoupling protein 1 (UCP1) following cold exposure or administration of a $\beta 3-\mathrm{AR}$ agonist (CL316,243) (9). Owing to the recent advance in the single-cell RNA sequencing

- Related Article: p. 247

Conflict of interest: The authors have declared that no conflict of interest exists.

Copyright: () 2020, American Society for Clinical Investigation.

Reference information: J Clin Invest. 2020;130(1):65-67. https://doi.org/10.1172/JCI133786.

(scRNA-seq) technology and inducible lineage-tracing methods, the field has gained a momentum to better understand the cellular heterogeneity in adipose tissues.

In this issue of the JCI, Song et al. set out to characterize the cellular heterogeneity in the interscapular BAT (10). First, the authors employed the Adipo-Chaser system, an inducible animal model in which all the differentiated adiponectin-expressing (Adipoq-expressing) adipocytes were permanently labeled as $\mathrm{LacZ}^{+}$cells following doxycycline administration $(10,11)$. The authors found that $\mathrm{LacZ}^{+}$brown adipocytes (Adipoq high-expressing cells) constituted $38 \%$ of total brown adipocytes in the BAT at room temperature $\left(24^{\circ} \mathrm{C}\right)$, whereas only $6 \%$ were $\mathrm{LacZ}^{+}$cells under a thermoneutral condition $\left(30^{\circ} \mathrm{C}\right)$. Notably, cold exposure robustly increased the number of $\mathrm{LacZ}^{+}$ cells $(76 \%$ of total brown adipocytes at $6^{\circ} \mathrm{C}$ ), although some cells remained LacZ(Adipoq low-expressing brown adipocytes). Further analyses by transmission electron microscopy showed that these $\mathrm{LacZ}^{+}$and LacZ ${ }^{-}$brown adipocytes were morphologically distinct in their lipid droplet size and mitochondrial contents (Figure 1). The authors next investigated whether the differences in the percentage of $\mathrm{LacZ}^{+}$cells involve de novo adipogenesis or cell death. When mature adipocytes were derived from prelabeled mice that were maintained at $24^{\circ} \mathrm{C}$ then subsequently pulsechased at $6^{\circ} \mathrm{C}$ or $30^{\circ} \mathrm{C}$, the percentages of $\mathrm{LacZ}^{+}$brown adipocytes were comparable with mice that were consistently held at $24^{\circ} \mathrm{C}$. Also, apoptosis signaling remained unchanged. These data suggest that there are two types of brown adipocytes, such that adiponectin-high and adiponectin-low cells in the BAT are interconvertible without cell death following acclimation to cold or thermoneutral conditions (10).

\section{Brown adipocytes with high versus low thermogenic capacities}

Is there any functional difference between the two forms of brown fat cells? To address 


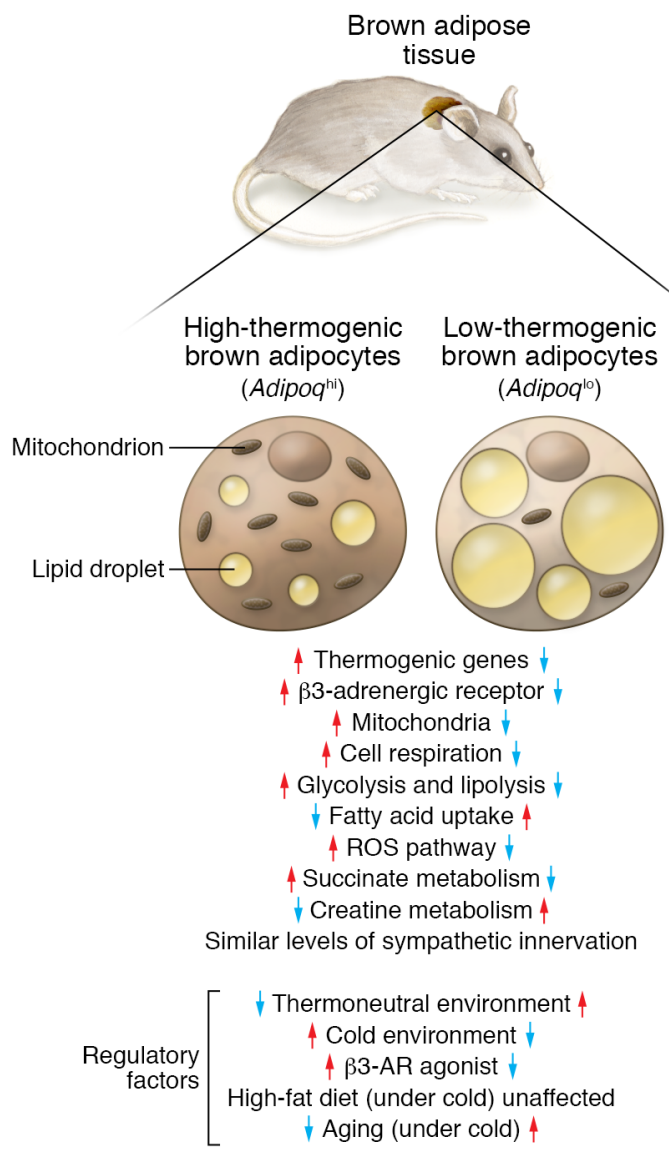

Figure 1. Two types of brown adipocytes in BAT. A population of brown adipocytes with low thermogenic activity and adiponectin expression coexists with brown adipocytes with high thermogenic activity and adiponectin expression.

this question, Song et al. performed scRNA-seq in primary brown adipocytes and identified 2352 Adipoq high-expressing cells and 1250 Adipoq low-expressing cells. In the Adipoq low-expressing cells, the authors found lower mRNA expression of genes related to oxidative phosphorylation, $\beta 3$-adrenergic receptor, lipolysis, glycolysis, fatty acid oxidation, and the TCA cycle relative to those of their high-expressing counterparts. Similarly, the Adipoq lowexpressing brown adipocytes expressed lower levels of genes involved in thermogenesis, reactive oxygen species (ROS), and succinate metabolism, as compared with the Adipoq high-expressing cells. On the other hand, the Adipoq low-expressing cells expressed higher levels of genes related to fatty acid uptake and UCP1-independent creatine futile cycle (12). Intriguingly, Adipoq high- and low-expressing cells disparately express two well-characterized master transcriptional regulators, Cebpa and Pparg, respectively, suggesting their roles in the transcriptional regulation of each cell type. Importantly, cell respiration was higher in Adipoq high-expressing cells relative to that in low-expressing cells. These data collectively suggest that the two populations of brown adipocytes have different cellular functions and metabolic profiles in that Adipoq high-expressing cells possess high thermogenic capacity while Adipoq low-expressing cells have low thermogenic capacity (10).

\section{Aging affects the cellular composition in BAT}

Given these distinct molecular features, the authors next aimed to address their regulatory pathways. Since BAT activity declines with obesity and aging $(13,14)$, the authors examined changes in the cellular composition of BAT by counting LacZ $^{+}$cells (Adipoq high-expressing cells) under a high-fat diet condition and in aged mice. The authors found that high-fat diet feeding failed to impair the cold-induced recruitment of Adipoq high-expressing brown adipocytes. On the other hand, the number of Adipoq high-expressing brown adipocytes declined gradually in aged mice (below $40 \%$ in 30-week-old and $20 \%$ in 60 -week-old mice) relative to young mice (approximately $70 \%$ in 10-week-old mice). These results indicate that the ability of BAT to recruit Adipoq high-expressing cells following cold stimuli declines with age (10).

In conclusion, the present study by Song et al. elegantly demonstrates the existence of two subpopulations of brown adipocytes that possess unique molecular and metabolic features (10). Do they arise from distinct progenitor pools? While embryonic brown adipocytes in the interscapular BAT arise from a $M y f 5^{+}$myogenic lineage (15), it remains unknown whether $M y f 5^{+}$cells persist as a progenitor source for Adipoq high-expressing and/or lowexpressing brown adipocytes in adult BAT. Another key question is: what are the mechanisms by which aging alters the cellular composition of BAT? A recent study reported that mitochondrial lipoylation in the BAT declines with age-associated impairment in thermogenesis and fuel oxidation. Of note, supplementation of $\alpha$-lipoic acids effectively restored BAT function, including thermogenesis and glucose oxidation, in aged mice (14). Thus, it would be interesting to test how $\alpha$-lipoic acid supplementation affects the cellular composition of Adipoq high- and low-expressing cells in aged BAT. A better understanding of the molecular mechanisms that underlie age-associated changes in BAT may lead to effective prevention or therapeutic measures for age-associated metabolic disorders, including obesity, insulin resistance, and type 2 diabetes.

\section{Acknowledgments}

We thank Zachary Brown for discussion and editorial suggestions. We also acknowledge support from the NIH (DK97441 and DK112268) and the Edward Mallinckrodt Jr. Foundation (to SK) and the Manpei Suzuki Diabetes Foundation (to YO).

Address correspondence to: Shingo Kajimura, 35 Medical Center Way, RMB1023, San Francisco, California 94143, USA. Phone: 415.476.9644; Email: shingo. kajimura@ucsf.edu. 
1. Kajimura S, Spiegelman BM, Seale P. Brown and beige fat: physiological roles beyond heat generation. Cell Metab. 2015;22(4):546-559.

2. Chondronikola $\mathrm{M}$, et al. Brown adipose tissue activation is linked to distinct systemic effects on lipid metabolism in humans. Cell Metab. 2016;23(6):1200-1206.

3. Bartelt A, et al. Brown adipose tissue activity controls triglyceride clearance. Nat Med. 2011;17(2):200-205.

4. Yoneshiro T, et al. BCAA catabolism in brown fat controls energy homeostasis through SLC25A44. Nature. 2019;572(7771):614-619.

5. Sidossis L, Kajimura S. Brown and beige fat in humans: thermogenic adipocytes that control energy and glucose homeostasis. J Clin Invest. 2015;125(2):478-486.

6. Sanchez-Gurmaches J, Guertin DA. Adipocytes arise from multiple lineages that are heterogeneously and dynamically distributed. Nat Commun. 2014;5:4099.

7. Sebo ZL, Rodeheffer MS. Assembling the adipose organ: adipocyte lineage segregation and adipogenesis in vivo. Development. 2019;146(7):dev172098.

8. Chen Y, et al. Thermal stress induces glycolytic beige fat formation via a myogenic state. Nature. 2019;565(7738):180-185.

9. Cinti S, et al. CL316,243 and cold stress induce heterogeneous expression of UCP1 mRNA and protein in rodent brown adipocytes. J Histochem Cytochem. 2002;50(1):21-31.

10. Song A, et al. Low- and high-thermogenic brown adipocyte subpopulations coexist in murine adipose tissue. J Clin Invest. 2020;130(1):247-257.
11. Wang QA, Tao C, Gupta RK, Scherer PE. Tracking adipogenesis during white adipose tissue development, expansion and regeneration. Nat Med. 2013;19(10):1338-1344.

12. Kazak L, et al. A creatine-driven substrate cycle enhances energy expenditure and thermogenesis in beige fat. Cell. 2015;163(3):643-655.

13. Yoneshiro T, et al. Age-related decrease in cold-activated brown adipose tissue and accumulation of body fat in healthy humans. Obesity (Silver Spring). 2011;19(9):1755-1760.

14. Tajima K, et al. Mitochondrial lipoylation integrates age-associated decline in brown fat thermogenesis. Nat Metab. 2019;1(9):886-898.

15. Seale P, et al. PRDM16 controls a brown fat/skeletal muscle switch. Nature. 2008;454(7207):961-967. 